\title{
Single Pion Measurement Capabilities at SciBooNE
}

\author{
Y. Nakajima for the SciBooNE collaboration \\ Department of Physics, Kyoto University, Kyoto 606-8502, Japan
}

\begin{abstract}
The precise knowledge of the single pion production cross-section of neutrino around the $\sim 1 \mathrm{GeV}$ energy region is an essential ingredient in the interpretation of neutrino oscillation experiments. The unique opportunities and prospects of single pion measurements at SciBooNE are described.
\end{abstract}

Keywords: neutrino, neutrino-nucleus cross-section

PACS: $01.30 . \mathrm{Cc}, 13.15 .+\mathrm{g}, 25.30 . \mathrm{Pt}$

\section{OVERVIEW}

The SciBooNE experiment[1] is a new neutrino experiment at FNAL aiming for precise cross-section measurements around the $\sim 1 \mathrm{GeV}$ energy region.

The detector is located on the Booster Neutrino Beamline (BNB) at Fermilab, which has been operated since 2002 for the MiniBooNE experiment[2]. The property of this beamline is well understood by MiniBooNE and also thanks to precise hadron production measurements by HARP[3]. The mean neutrino energy is $\sim 0.8 \mathrm{GeV}$, which is almost the same as that of T2K[4]. The detailed description of SciBooNE detector complex can be found elsewhere[1,5]. The main component, SciBar[6], is a fully active finely segmented tracking detector which previously used at $\mathrm{K} 2 \mathrm{~K}$. This combination of SciBar and the well understood high intensity BNB will give us great opportunities for precise cross-sections measurements around the $\sim 1 \mathrm{GeV}$. The cross-section data in this energy range will directly help T2K understand their neutrino flux and also MiniBooNE as a near detector.

\section{MOTIVATIONS TO STUDY SINGLE PION PRODUCTIONS}

Understanding the cross-section of charged current single pion production $\left(\mathrm{CC} 1 \pi^{+}=\right.$ $v_{\mu} N \rightarrow \mu^{-} N \pi^{+}$, where $\mathrm{N}=\mathrm{p}, \mathrm{n}$ ) is especially important to study $v_{\mu} \rightarrow v_{X}$ oscillation, which will be made by, for example, T2K and MiniBooNE. These experiments utilize Cherenkov type detectors for neutrino detection and the signal event will be generated by muon from charged current quasi elastic scattering (CCQE) because it is possible to reconstruct the energy of neutrino from observed muon. However, this measurement suffers from large number of backgrounds which dominantly come from $\mathrm{CC} 1 \pi^{+}$interactions. This is because the pion is not observed since its momentum is below the Cherenkov threshold. Moreover, the pion can be absorbed in nuclei, making the distinction between signals and backgrounds even harder to accomplish. 
In the case of $\mathrm{T} 2 \mathrm{~K}$, the $\mathrm{CC} 1 \pi^{+} / \mathrm{CCQE}$ cross-section ratio is required to be understood at $5 \%$ level to keep the resulting error on the oscillation parameters comparable to that due to statistical uncertainties[1]. However, the past cross-section measurements have been limited by low statistics and large systematic uncertainty. Therefore, precise measurements are strongly needed. Recently, K2K and MiniBooNE are starting to make precision measurements on $\mathrm{CC} 1 \pi^{+}$cross-section[7, 8], and SciBooNE will follow with higher statistics than $\mathrm{K} 2 \mathrm{~K}$ and better resolution than MiniBooNE.

\section{$\operatorname{CC1} \pi^{+}$MEASUREMENT AT SCIBOONE}

\section{Detector Performance}

One of the most important features of the SciBar detector is that every charged particle including the protons and pions can be reconstructed as tracks. Figure 1 shows Monte Carlo event displays for $\mathrm{CC} 1 \pi^{+}$interactions occurring in the SciBar detector.

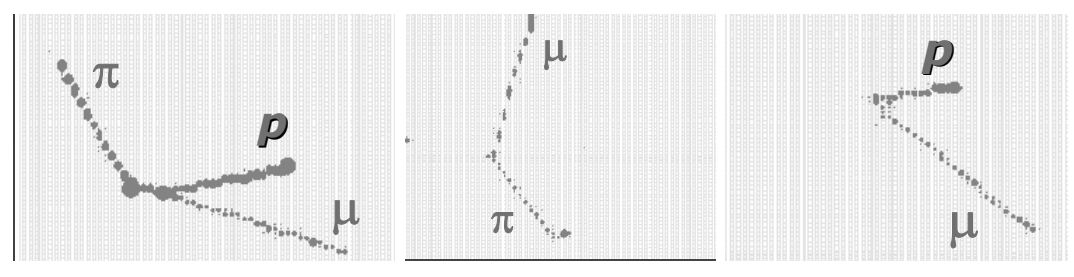

FIGURE 1. Event displays of typical Monte Calro $\mathrm{CC} 1 \pi^{+}$interactions.

The event display on the left is a typical $\nu_{\mu} p \rightarrow \mu^{-} p \pi^{+}$event. We can see the tracks of all charged particles and also can clearly distinguish the muon/pion tracks from the proton track by their energy depositions. So we can clearly identify such an event with two MIP-like tracks and a proton-like track.

Also, we can distinguish $\nu_{\mu} n \rightarrow \mu^{-} n \pi^{+}$from $\nu_{\mu} p \rightarrow \mu^{-} p \pi^{+}$by the existence of a proton track. The center figure shows a typical $\nu_{\mu} n \rightarrow \mu^{-} n \pi^{+}$event. Furthermore, even if the pion is absorbed in the nucleus, we can distinguish $\mathrm{CC} 1 \pi^{+}$from CCQE by using proton and muon kinematics. The right figure is an example of $\nu_{\mu} p \rightarrow \mu^{-} p \pi^{+}$where the $\pi^{+}$is absorbed.

This clear event-by-event final state tagging should enables us to unambiguously identify $\mathrm{CC} 1 \pi^{+}$event, and also to study kinematical characteristic such as pion momentum distribution or pion absorption cross-section.

\section{Current Status and Expected Sensitivity}

We completed detector construction and installation in April 2007. Then after the detector commissioning using cosmic rays, we started the commissioning using the BNB at the end of May 2007, and we successfully detected (anti-)neutrino events. Figure 2 is an event display of a $\stackrel{(-)}{\vee} \mu p \rightarrow \mu^{ \pm} p \pi^{\mp}$ candidate, which we took in the early stage of the commissioning. One can clearly see two MIP-like tracks which come from a muon and a pion, and the vertex activity which results from a proton. 


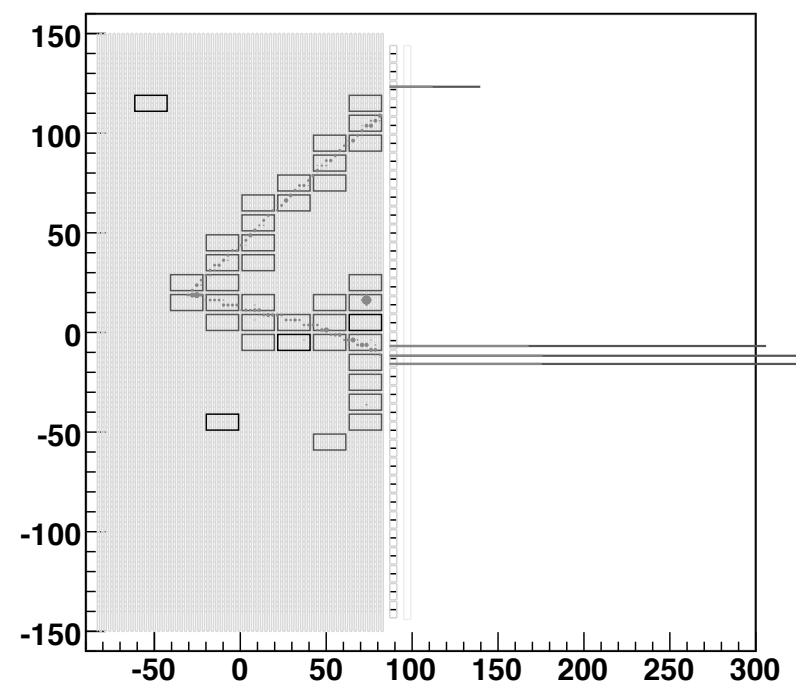

FIGURE 2. An event display of a $\stackrel{(-)}{v}_{\mu} p \rightarrow \mu^{ \pm} p \pi^{\mp}$ candidate.

The commissioning phase allowed us to confirm that the detectors are working as expected and we are collecting (anti-)neutrino data. It is expected to collect $\sim 28000$ $\mathrm{CC} 1 \pi^{+}$interactions with $1 \times 10^{20}$ protons on target for neutrino mode. This allows us to measure $\mathrm{CC} 1 \pi^{+} / \mathrm{CCQE}$ cross-section ratio with less than $5 \%$ statistical uncertainty, which will provide sufficient input for $v_{\mu}$ disappearance measurements at T2K and MiniBooNE.

\section{ACKNOWLEDGMENTS}

SciBooNE collaboration gratefully acknowledges support from various grants and contracts from the Department of Energy (U.S.), the National Science Foundation (U.S.), the MEXT (Japan), the INFN (Italy) and the Spanish Ministry of Education and Science. The author was supported by Japan Society for the Promotion of Science.

\section{REFERENCES}

1. SciBooNE Collaboration, arXiv:hep-ex/0601022.

2. A. A. Aguilar-Arevalo et al. [The MiniBooNE Collaboration], Phys. Rev. Lett. 98, 231801 (2007) [arXiv:0704.1500 [hep-ex]].

3. HARP Collaboration, arXiv:hep-ex/0702024v2.

4. T2K Collaboration, arXiv:hep-ex/0106019.

5. R. Napora, In these proceedings.

6. K. Nitta et al., Nucl. Inst. Meth. A 535 (2004) 147.

7. B. Fleming, In these proceedings.

8. L. Whitehead, In these proceedings. 